\title{
SEL1L deficiency impairs growth and differentiation of pancreatic epithelial cells
}

\author{
Shuai Li ${ }^{1}$, Adam B Francisco ${ }^{1}$, Robert J Munroe ${ }^{2}$, John C Schimenti ${ }^{2}$, Qiaoming Long ${ }^{1 *}$
}

\begin{abstract}
Background: The vertebrate pancreas contains islet, acinar and ductal cells. These cells derive from a transient pool of multipotent pancreatic progenitors during embryonic development. Insight into the genetic determinants regulating pancreatic organogenesis will help the development of cell-based therapies for the treatment of diabetes mellitus. Suppressor enhancer lin12/Notch 1 like (Sel11) encodes a cytoplasmic protein that is highly expressed in the developing mouse pancreas. However, the morphological and molecular events regulated by Sel11 remain elusive.

Results: We have characterized the pancreatic phenotype of mice carrying a gene trap mutation in Sel11. We show that Sel1/ expression in the developing pancreas coincides with differentiation of the endocrine and exocrine lineages. Mice homozygous for the gene trap mutation die prenatally and display an impaired pancreatic epithelial morphology and cell differentiation. The pancreatic epithelial cells of Sel1/ mutant embryos are confined to the progenitor cell state throughout the secondary transition. Pharmacological inhibition of Notch signaling partially rescues the pancreatic phenotype of Sel1/ mutant embryos.
\end{abstract}

Conclusions: Together, these data suggest that Sel1/ is essential for the growth and differentiation of endodermderived pancreatic epithelial cells during mouse embryonic development.

\section{Background}

The multiple cell types that make up the adult pancreas, including endocrine, exocrine and ductal cells, derive from a common pool of pancreatic progenitors. Pancreatic development in mice begins at embryonic day 9.5 (E9.5) with the formation of two epithelial buds on the dorsal and ventral side of the primitive gut endoderm [1]. Epithelial cells within the pancreatic buds proliferate rapidly and branch out during later embryonic days to form a complex tubular network comprised of undifferentiated multipotent progenitor cells $[2,3]$. Starting at E13.5, the expanded pancreatic epithelial cells undergo an asynchronized wave of differentiation to give rise to all the differentiated cell types of the adult pancreas, including acinar cells that produce hydrolytic digestive enzymes and islet cells that secrete endocrine hormones [4,5]. Pancreatic morphogenesis depends on a complex and yet incompletely characterized network of transcription factors. Significant efforts have been made

\footnotetext{
* Correspondence: q139@cornell.edu

'Department of Animal Science, College of Agriculture and Life Sciences,
} Cornell University, Ithaca, NY 14850, USA in the past few years to understand the role of several important transcription factors, including $P d x 1[6,7]$, Ptf1a [8,9], Sox9 [10,11]9, Ngn3 [12,13], NeuroD1 [14,15], Pax4 [16], Pax6 [17], Nkx2.2 [18], Nkx6.1 [19], Arx [20], Isl1 [21] and Insm1 [22]. It is generally accepted that these transcription factors coordinate pancreatic morphogenesis by functioning in concert to restrict the developmental potentials of the pancreatic progenitors in a spatial and stage-specific manner [23].

Several previous studies have underscored the importance of Notch-mediated signaling in regulating pancreatic cell proliferation and cell fate decisions through control of Ngn3 gene expression. During pancreatic development, $\mathrm{Ngn} 3$ is transiently expressed in a subset of the pancreatic epithelial cells. NGN3 deficiency completely abolishes formation of all the endocrine cell subtypes, suggesting Ngn3 functions as a master switch for the endocrine lineage in the pancreas. Mutations in genes encoding Notch signaling pathway components, such as DLL1 (ligand), RBP-Jk (the intracellular mediator), or HES-1 (the effector) causes expansion of Ngn3 expression in pancreatic cells and, as a result, accelerated 
differentiation of endocrine cells at the expense of acinar and ductal cells $[13,24,25]$. Conversely, over or persistent expression of the Notch intracellular domain (NICD), a constitutively active form of Notch receptors, or the Notch effector gene Hes1 results in diminished expression of $\mathrm{Ngn} 3$ and attenuated differentiation of endocrine cells [26-28]. These studies suggest that during pancreatic development Notch signaling controls the endocrine and exocrine cell fate decisions of pancreatic epithelial cells by directly regulating Ngn3 expression. Recent studies have also indicated the importance of Notch signaling in control of exocrine cell differentiation. Ectopic expression of activated NOTCH-1 prevents or significantly delays differentiation of acinar cells [26,27].

While the role of Notch signaling in control of pancreatic cell proliferation and cell fate decisions is clearly recognized, the molecular mechanisms necessary for proper control of Notch signaling during vertebrate pancreatic development are poorly understood. Genetic and biochemical studies in invertebrates suggest that regulation of Notch signaling occurs at various levels and through multiple mechanisms [29-31]. These include stochastic and/or developmental expression of the Notch receptors and their ligands [32-34], selective receptor-ligand interactions [35,36], intracellular protein trafficking [37] and stability of NICD [38]. Suppressor enhancer lin12 1 like (Sel1l) encodes a cytoplasmic protein that is highly conserved throughout the vertebrate kingdom [39]. RNA in situ hybridization and immunohistological analysis revealed that Sel1l is highly expressed in both the embryonic and adult pancreas [40-42]. The human Sel1l gene is located in a chromosome region that is in close proximity to a type 1 diabetes high risk locus, IDDM-11 (insulin-dependent diabetes mellitus locus 11), prompting the speculation that mutations in Sel1l may be associated with the pathogenesis of type 1 diabetes [43]. Sel-1, the C. elegans ortholog of Sel1l, was first identified in a genetic screen for mutations that suppress lin-12/Notch activity [44]. Subsequent biochemical studies demonstrated that Sel-1 negatively regulates lin-12/Notch activity by controlling lin-12/Notch turn-over $[45,46]$. Based on these findings, it has been suggested that Sel1l may also function as a negative regulator for Notch signaling [47]. Recent biochemical and molecular studies in vitro revealed that Selll is also required for maintaining homeostasis of the endoplasmic reticulum (ER). SEL1L nucleates an ER membrane protein complex that is required for dislocation of unfolded or misfolded proteins from the ER lumen into the cytosol for degradation [48,49].

In an attempt to decipher the developmental and physiological roles of Sel1l, we have generated and characterized mice carrying a gene trap mutation in the Sel1l gene. We report here that Sel1l expression during pancreas development coincides with differentiation of pancreatic epithelial cells into both the endocrine and exocrine lineages. Homozygous Sel1l mutant embryos exhibit an impaired pancreatic epithelial growth and branching morphology. Pharmacological inhibition of Notch signaling rescues the pancreatic phenotype of Sel1l-deficient embryos. These data are consistent with the notion that Selll functions as a negative regulator for Notch signaling during pancreatic organogenesis [47].

\section{Results}

Sel1/ expression during mouse pancreatic development

We generated mice carrying a gene-trap insertion in the Sel1l gene. The gene trap cassette, located in intron 14, contains a $\beta$-galactosidase-neomycin ( $\beta$ geo) fusion reporter gene. To determine the spatiotemporal expression pattern of Sel1l in the developing mouse pancreas, we performed immunohistological analysis of pancreatic sections from Sel11 $l^{+/ \beta g e o}$ embryos using antibodies against $\beta$-galactosidase ( $\beta$ gal) and several pancreatic proteins, including SOX9 and PDX1 (progenitor markers), insulin and glucagon (endocrine lineage markers) and amylase (exocrine lineage marker). $\beta$ gal expression was first detected at E12.5 in a small number of cells located within the core of SOX9-expressing pancreatic epithelium (Fig. 1A-B). The $\beta_{g a l}{ }^{+}$cells at E12.5 express either glucagon (Fig. 1C) or insulin (Fig. 1D), indicating that they were early endocrine cells. $\beta$ gal expression expands significantly between E12.5 and E14.5. At E14.5, differential $\beta$ gal expression was detected in the $\mathrm{PDX}^{+}$cells throughout the pancreatic epithelium (Fig. 1E). Epithelial cells with markedly lower $\beta$ gal expression correspond to a subset of PDX $1^{+}$cells that express SOX9 (Fig. 1F). At E15.5, high $\beta$ gal expression was detected in the core of the differentiating pancreas (Fig. 1G-H, asterisks). These $\mathrm{Bgal}^{+}$cells were positive either for glucagon or insulin (arrows in Fig. 1I and 1J, respectively). At birth, $\beta$ gal expression was detected in both islet and acinar cells (Fig. 1K-L, asterisk and arrow, respectively). Taken together, these expression data indicate that Sel1l is selectively expressed in the differentiating or differentiated pancreatic epithelial cells.

\section{SEL1L deficiency results in defective pancreatic epithelial growth and branching morphogenesis}

The gene trap in Sel1l contains a strong splicing acceptor signal that efficiently blocks splicing between exon 14 and 15 , resulting in a truncated Sel1l transcript. Homozygous gene trap mice $\left(\mathrm{Sel}_{1} l^{\beta g e / \beta g e o}\right)$ exhibit systemic endoplasmic reticulum stress and die before E13.5 to E15.5 (Additional file 1). To determine if Sel1l is required for pancreatic epithelial growth, we performed morphometrical analysis of the developing pancreas of viable Sel1 $l^{\beta g e o / \beta g e o}$ embryos. At E11.5, the dorsal and 

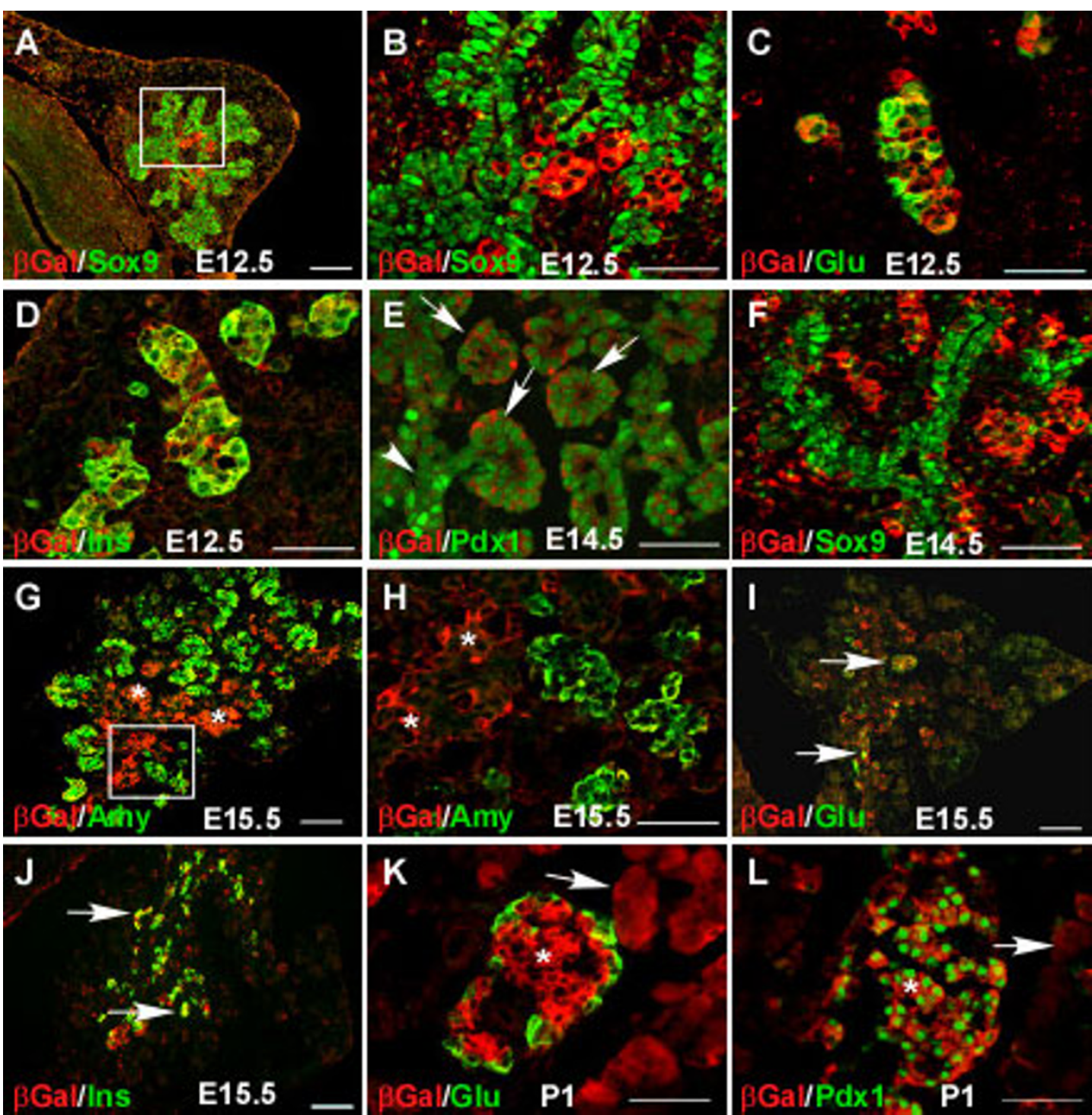

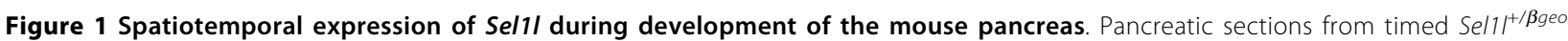
embryos were co-immunostained with antibodies against $\beta \mathrm{Gal}$ (red) and various pancreatic markers (green) as indicated on the left side of each panel. (B and $\mathbf{H}$ ) Magnified views of the boxed area in $\mathbf{A}$ and $\mathbf{G}$, respectively. (A-D) $\beta$ Gal expression at E12.5. $\beta$ Gal expression begins in a small number of cells within the core pancreatic epithelium at E12.5 (A). At this stage, there is no co-expression of $\beta$ Gal and SOX9 (B); however, $\beta$ Gal is co-expressed with either glucagon (C) or insulin (D). (E-F) $\beta$ Gal expression at E14.5. $\beta$ Gal is differentially expressed in the PDX $1^{+}$cells (E). While high $\beta \mathrm{Gal}$ signal was detected in the epithelial branches (arrows), no or very low Gal signal was detected in the core "duct-like" epithelium (arrowhead). These core epithelial cells correspond to the subset of PDX ${ }^{+}$cells that express SOX9 (F). (G-J) $\beta$ Gal expression at E15.5. $\beta$ Gal expression increases markedly in the core pancreatic epithelium ( $\mathbf{G}$ and $\mathbf{H}$, asterisks). $\beta$ Gal co-localizes with either glucagon (I, arrows) or insulin (J, arrows). (K-L) $\beta$ Gal expression at postnatal day 1. $\beta$ Gal is expressed in both islet and acinar tissues (K, asterisk and arrow, respectively). $\beta$ Gal co-localizes with PDX1 in the islet tissue (L). Scale bars: (A, G, I and $\mathbf{J}) 50 \mu \mathrm{m} ;(\mathbf{B}-\mathbf{F}, \mathbf{K}-\mathbf{L}) 100 \mu \mathrm{m}$.

ventral pancreatic buds in Sel1 $1 l^{\beta g e o / \beta g e o}$ embryos appeared to be closely linked (Fig. 2B), as compared to those in wild-type embryos (Fig. 2A). There was no significant difference in the pancreatic epithelial size between wild-type and Sel1 $l^{\beta g e o / \beta g e o}$ embryos (Fig. 2E). At E13.5, the dorsal pancreatic bud of Sel11 $l^{\text {Bgeo/ } / \text { ggeo }}$ embryos (Fig. 2C) was clearly smaller than that of wildtype embryos (Fig. 2F). In addition, while wild-type pancreatic epithelium displayed a well-branched structure, mutant pancreatic epithelium appeared to be a simple tube of epithelial cells. TUNEL assays revealed no significant increase of apoptosis in the developing pancreas of Sel1l mutant embryos (data not shown). Immunostaining using anti-PCNA (a cell proliferation indicator) antibody indicated that the pancreatic epithelium of Sel1l mutant embryos had a significantly lower 
rate of cell proliferation than wild-type pancreatic epithelium (Fig. 2G). Together, these results indicate that $\mathrm{Sel} 1 \mathrm{l}$, while dispensable for pancreatic epithelial induction, is essential for the subsequent growth and branching morphogenesis of the pancreatic epithelial cells.
SEL1L deficiency inhibits differentiation of acinar cells and significantly attenuates differentiation of endocrine cells We next investigated the role of Sel1l in pancreatic epithelial cell differentiation. Since the first wave of cell differentiation occurs before E12.5 and the cells generated are mostly $\alpha$-cells, we analyzed glucagon expression
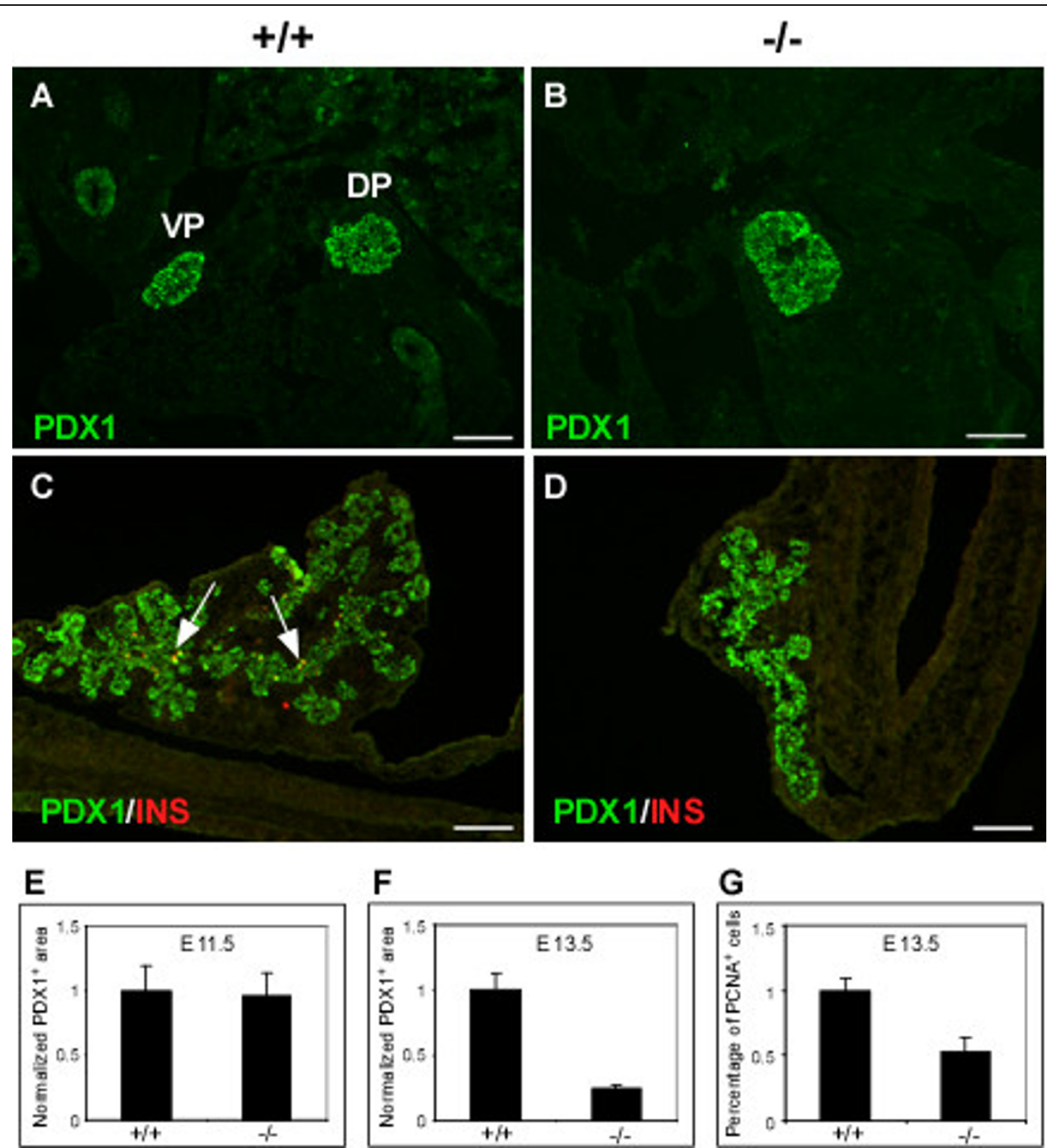

Figure 2 Impaired pancreatic epithelial growth and branching morphogenesis in Sel1/ ${ }^{\beta \text { geo } / \beta \text { geo }}$ embryos. (A-D) Immunohistological analysis of the developing pancreas of wild-type and Sel1 ${ }^{\beta g e o / \beta g e o}$ embryos at E11.5 and E13.5; the genotypes of the pancreatic sections are indicated as +/+ and -/-, respectively. The following antibodies were used: Pdx1 (A-D, green) and insulin (C-D, red). (A-B) The dorsal and ventral

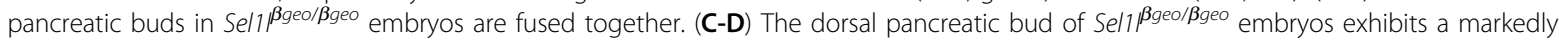
reduced epithelial size and an impaired branching morphology. (E-F) Statistical analyses of estimated pancreatic epithelial sizes of wild-type and

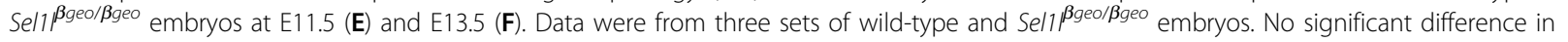
the epithelial sizes of wild-type and Sel11-deficient pancreas at E11.5 was detected (E). At E13.5, the pancreatic epithelium of Sel11-deficient embryos was significantly smaller (F). Scale bar: $100 \mu \mathrm{m}$. 
in E11.5 Sel1 $l^{\beta g e o / \beta g e o}$ embryos. As shown in Fig. 3A-B and 3G, comparable numbers of glucagon ${ }^{+}$cells were observed in wild-type and Sel1 $l^{\text {Bgeo/Rgeo }}$ embryos. Sel1l is thus unlikely to be required for the generation of early endocrine cells during the first transition (E9.5 to E12.5).

While the majority of Sel1 $l^{\text {Bgeo/Rgeo }}$ embryos (95\%) die before the initiation of the major wave of cell differentiation in the developing pancreas (E13.5), about 5\% of these embryos are viable at E15.5. To determine if Sel1l is required for differentiation of pancreatic epithelial cells during the secondary transition (E13.5 to E15.5), we analyzed the expression of three pancreatic lineage markers: insulin ( $\beta$-cells), glucagon ( $\alpha$-cells) and amylase (acinar cells) in these embryos. Significant numbers of glucagon $^{+}$and insulin ${ }^{+}$cells were detected in wild-type embryos at E15.5 (Fig. 3C and 3E). In contrast, the numbers of glucagon ${ }^{+}$and insulin ${ }^{+}$cells were significantly reduced in Sel1 $l^{\beta \text { geo/ } / \text { ggeo }}$ embryos (Fig. 3D and 3F, $3 \mathrm{H}$ and $3 \mathrm{I})$. Abundant amylase ${ }^{+}$cells are present in wild-type embryos at E15.5 (Fig. 4E). In contrast, no amylase $^{+}$cells are detectable in Sel1 $l^{\beta g e o / \beta g e o}$ embryos (Fig. 4F). Disruption of Sel1l function thus results in an impaired differentiation of both acinar and islet cells.

\section{SEL1L-deficient pancreatic epithelium exhibits inhibited growth and differentiation ex vivo}

To ensure that the growth and differentiation defects observed in Sel1 $l^{\beta g e o / \beta g e o}$ embryos were not due to the effect of global embryonic growth retardation of these embryos, we studied the pancreatic phenotype of Sel1 $l^{\beta g e o /}$ $\beta g e o$ embryos using an organ culture system. The dorsal pancreatic bud of wild-type and Sel1 $1^{\beta g e o / \beta g e o}$ embryos were isolated at E11.5 and cultured for 8 days. The cultured pancreatic explants were then analyzed by immunofluorescence using antibodies against PDX1 and insulin. In general, Selll-deficient pancreatic epithelium grows poorly (Suppl. Fig. 2B and 2D) with about half of the cultured mutant epithelia failing to form branched epithelial structures. This is in sharp contrast to the growth behavior of wild-type pancreatic epithelia, which form a branched epithelial structure (Additional file 2). The number of insulin $^{+}$cells was significantly decreased in Selll-deficient pancreatic explants (Fig. 4B), as compared to that in wildtype control explants (Fig. 4A). Amylase ${ }^{+}$cells were detected in cultured Sel1l-deficient explants (Fig. 4D), although the number of amylase ${ }^{+}$cells was significantly lower compared to that in wild-type control explants (Fig.

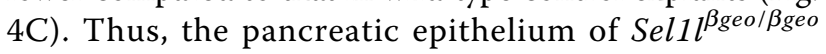
embryos displays impaired growth, branching morphogenesis and lineage differentiation ex vivo. These findings confirm that the pancreatic defects observed in Sel1l-deficient embryos at E15.5 are not due to the global growth retardation.
Pancreatic progenitor cells in SEL1L-deficient embryos at E15.5 are confined to a pluripotent progenitor state

To gain insight into the molecular basis underlying the pancreatic defects in Sel1 ${ }^{\beta g e o / \beta g e o}$ embryos, we first analyzed the expression of several transcription factors important for pancreatic epithelial growth and lineage formation. Immunostaining was carried out to assess the expression of Sox9, Pdx1 and Ptf1a in E15.5 Sel1 ${ }^{\text {Bgeo/ßgeo }}$ embryos. PDX1 expression was uniformly detected in the pancreatic epithelial cells of Sel1 $l^{\beta g e o / \beta g e o}$ embryos (Fig. 5B). This is in sharp contrast to wild-type embryos where PDX1 expression was differentially expressed in the pancreatic epithelial cells, with high expression in a subset of the core epithelial cells and low expression in the peripheral epithelial cells (Fig. 5A, white arrow and red arrowheads, respectively). No PTF1a expression was detected in the pancreatic epithelium of Sel1 $l^{\beta g e o / \beta g e o}$ embryos (Fig. 5B), whereas clear co-expression of PTF1a and PDX1 was detected in the proacini in the periphery of the pancreatic epithelial buds (Fig. 5A, white arrows). SOX9 was detected in approximately $20 \%$ of the PDX $1^{+}$ cells in wild-type embryos (Fig. 5C, red arrows). In contrast, SOX9 expression was observed in more than $70 \%$ of the PDX1 ${ }^{+}$cells in Sel1 $1^{\beta g e o / \beta g e o}$ embryos (Fig. 5D, asterisk). These findings are consistent with the notion that the pancreatic epithelial cells in Sel1 $l^{\text {Bgeo/ } / \text { ggeo }}$ embryos at E15.5 are restricted the pancreatic progenitor state.

\section{Pharmacological inhibition of Notch signaling rescues endocrine lineage formation in Sel1 ${ }^{\beta g e o / \beta g e o}$ embryos} Sel1l was thought to be a negative regulator for Notch signaling [47]. Indeed, the pancreatic phenotype in Sel1 $l^{\text {pgeo/ }}$ $\beta$ geo embryos shows remarkable similarity to that of mouse or zebrafish embryos over-expressing Notch intracellular domain, the constitutively active form of Notch receptors $[26,28,50]$. We speculated that the impaired pancreatic epithelial growth and differentiation in Sel11-deficient embryos may be due to an increased Notch signaling activity. To test this possibility, we used the $\gamma$-secretase inhibitor, DAPT (Difluorophenacetyl-al-alanyl-S-phenylglycine-t-butyl ester), to suppress Notch signaling in pancreatic explants culture. The dorsal pancreatic bud of wild-type and $\mathrm{Sel} 1 l^{\beta \text { geo/ßgeo }}$ embryos was isolated at E11.5 and cultured for 8 days in the absence or presence of DAPT. At a concentration of $10 \mu \mathrm{M}, \mathrm{DAPT}$ did not exhibit detectable effects on the growth, branching morphology and endocrine cell differentiation of wild-type pancreatic epithelium (Fig. 6A-B and 6E). At the same concentration, however, DAPT caused a significant expansion of the SEL1L-deficient pancreatic epithelium (Fig. 6C-D). Importantly, DAPT treatment resulted in a marked increase in the number of insulin ${ }^{+}$cells (Fig. 6D and 6F). These observations indicate that pharmacological 


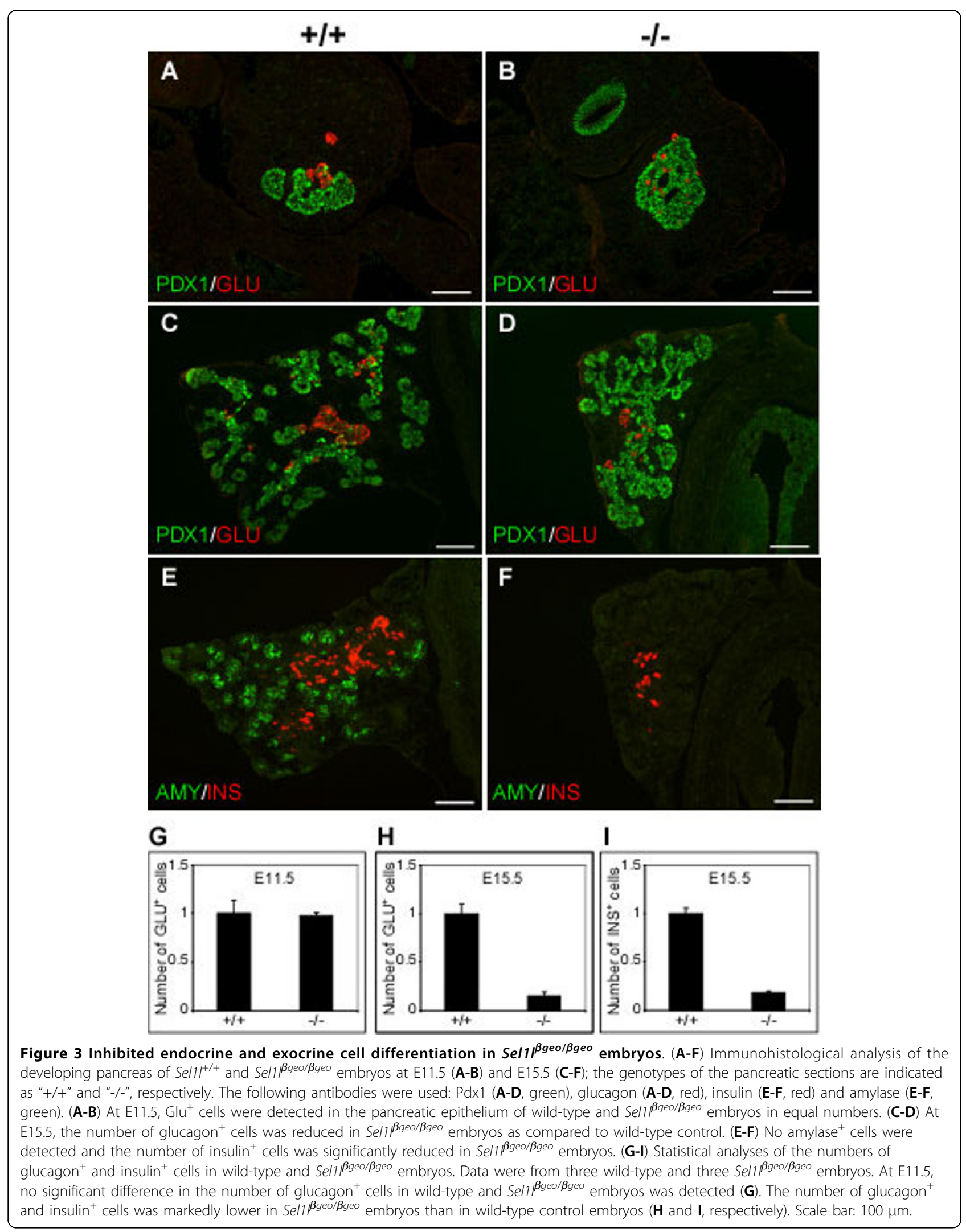




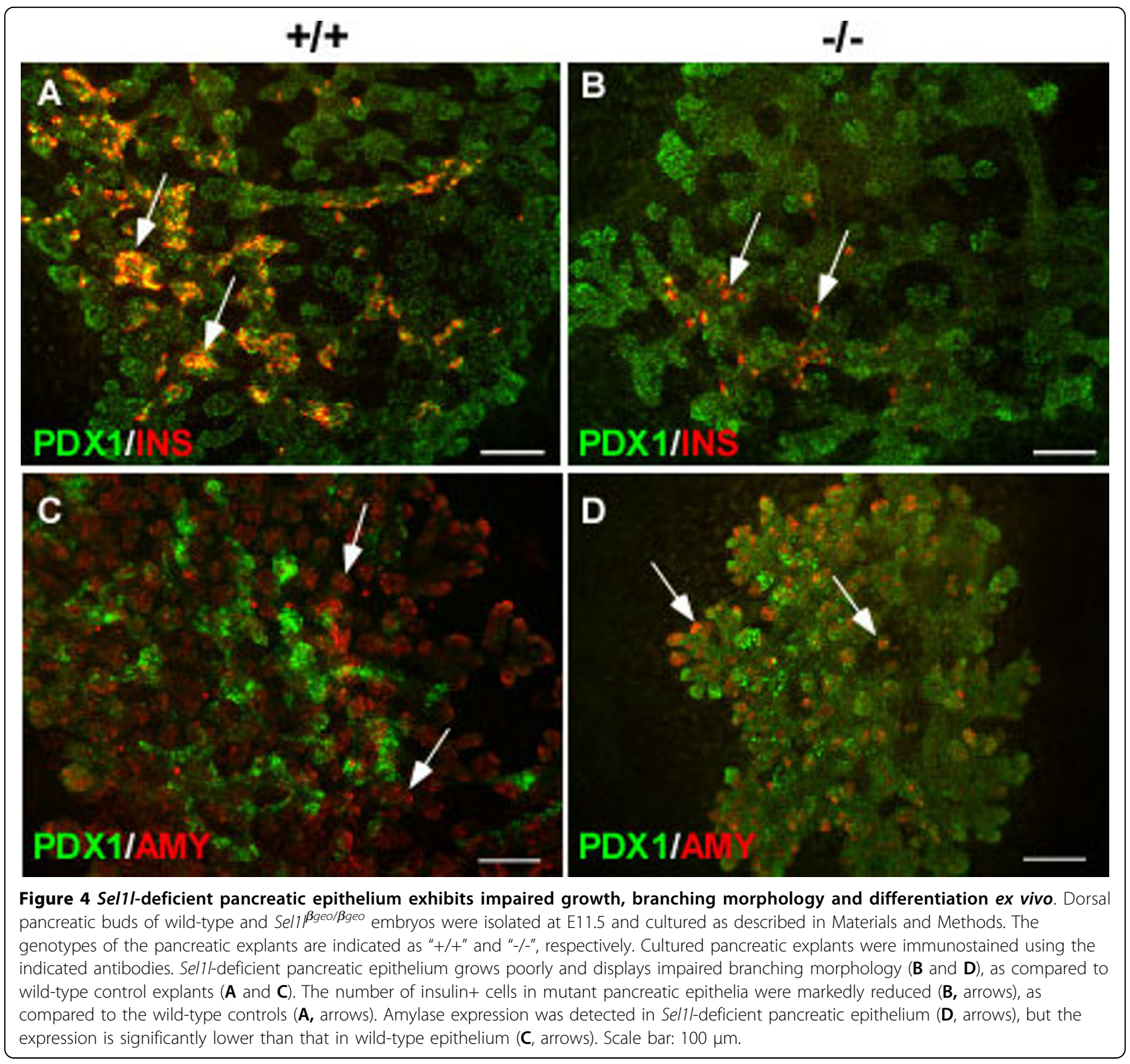

inhibition of Notch signaling rescues, at least partially, the pancreatic epithelial growth and endocrine differentiation defects of $\operatorname{Sel1} l^{\beta g e o / \beta g e o}$ embryos.

\section{Discussion}

The genetic determinants underlying vertebrate pancreatic development are not completely understood. In the present study, we report that Selll expression in the developing mouse pancreas coincides with differentiation of the endocrine and exocrine lineages during the second transition. Embryos homozygous for a gene trap insertion in Sel1l display impaired pancreatic epithelial growth and differentiation. Pharmacological inhibition of Notch signaling partially rescues the pancreatic phenotype of SEL1L-deficient embryos. Together, these data provide evidence that Sel1l is required for growth and differentiation of the mammalian pancreatic epithelial cells. The underlying mechanism may be that Sel1l functions as a negative regulator for the Notch signaling pathway by facilitating degradation of the Notch intracellular domain.

Donoviel et. al. previously reported that the mouse Sel1l gene is highly transcribed in the acini of the developing pancreas at E14.5 and E17.5 [41]. We show, by immunohistological analysis of the $\beta$-galactosidase reporter in Sel1l, that Selll is also highly expressed in early glucagon ${ }^{+}$and insulin ${ }^{+}$cells (Fig. 1C and 1D, respectively) generated during the first transition period 


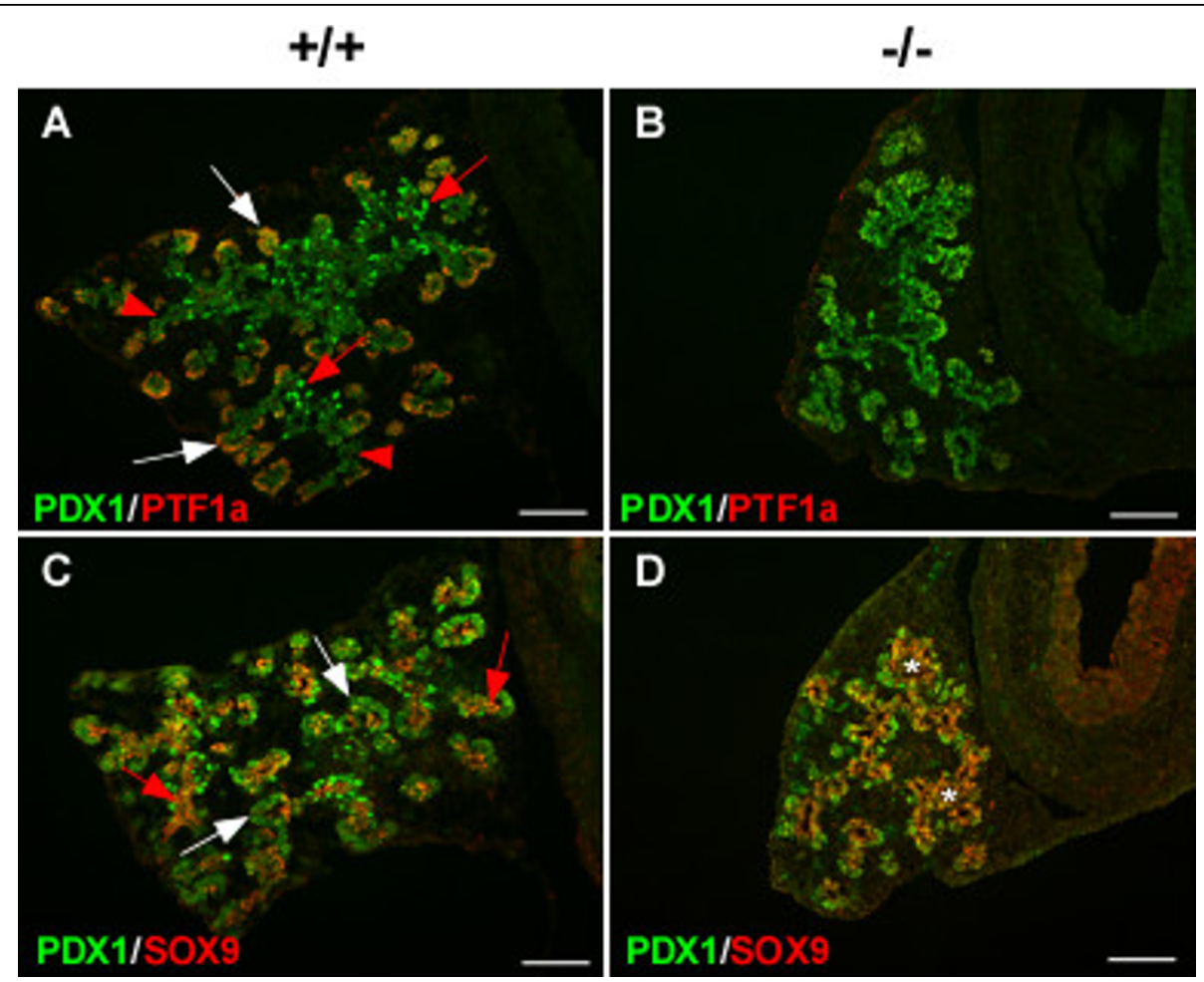

Figure 5 Sel1/-deficient pancreatic epithelial cells fail to commit to lineage precursors during the secondary transition. (A-D)

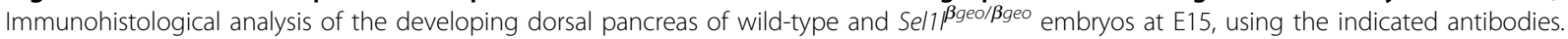
The genotypes of the pancreatic sections are indicated as " $+/+^{\prime \prime}$ and " $-I^{-}$", respectively. (A-B) PTF1a is expressed in the periphery of wild-type pancreatic epithelium marking the commitment of pancreatic epithelial cells into the exocrine lineage (A, white arrows); PTF1a expression is absent in the mutant pancreas (B). Up-regulation of PDX1 expression in a subset of epithelial cells in the core of wild-type pancreatic epithelium marks the commitment of pancreatic epithelial cells into the endocrine lineage (A, red arrows); whereas PDX1 is uniformly expressed in mutant pancreatic epithelial cells (B). (C-D) SOX9 is expressed in a small population of epithelial cells in wild-type pancreatic epithelium (C, red arrows); in contrast, SOX9 mostly co-localizes with PDX1 in mutant pancreatic epithelium (D, asterisks). Scale bar: $100 \mu \mathrm{m}$.

of pancreatic development $[5,51,52]$. Consistent with the Sel1l mRNA expression pattern reported by Donoviel et. al., we reveal that at E14.5 Sel1l is broadly expressed in the $\mathrm{PDX}^{+}$pancreatic epithelia (Fig. 1E). Interestingly, significantly lower Sel1l expression is observed in the undifferentiated pancreatic epithelial cells (Fig. 1F) that express SOX9 $[10,11]$. Our findings therefore indicate that Sel1l expression is enhanced in the committed pancreatic epithelial cells or differentiated endocrine and exocrine cells.

The selective expression of Sel1l in differentiating or differentiated pancreatic epithelial cells is highly suggestive of a role for Sel1l in promoting lineage differentiation during the secondary transition. To define the role of Sel1l in pancreatic development, we characterized the pancreatic phenotype of embryos homozygous for a gene trap insertion in Sel1l. We show that wild-type and Sel1ldeficient embryos have a comparable pancreatic epithelial size at E11.5 (Fig. 2A-B and 2E), suggesting that Sel1l function is dispensable for the induction and early growth of pancreatic epithelial cells. At E13.5, however,
Sel1l-deficient pancreatic epithelia are significantly smaller and display an impaired branching morphology (Fig. $2 \mathrm{C}-\mathrm{D}$ and 2F). Importantly, Sel1l-deficient pancreatic epithelia at E15.5 completely lack amylase ${ }^{+}$cells (Fig. 3F) and have significantly reduced numbers of glucagon ${ }^{+}$and insulin $^{+}$cells (Fig. 3D and 3F, respectively). Also, by analyzing the phenotype of cultured Sel1l-deficient dorsal pancreatic epithelia, we confirmed that the pancreatic defects in Sel1l-deficient embryos are not due to the effect of global growth retardation of the Sel1l-deficient embryos (Fig. 4A-D). Together, these data strongly suggest that Sel1l plays an important role in mouse pancreatic organogenesis by facilitating differentiation of the endocrine and exocrine lineages of the pancreas.

The C. elegans ortholog of the mammalian Sel1l gene, Sel-1, was originally identified by genetic screening as a negative regulator for lin-12/Notch signaling $[44,53]$. Biochemical studies have shown that Sel-1 regulates lin-12/ Notch receptor turnover $[45,46]$. Whether or not Sel11 has similar functions in the mammalian system remains unclear [54]. In this study, we provide two lines of 


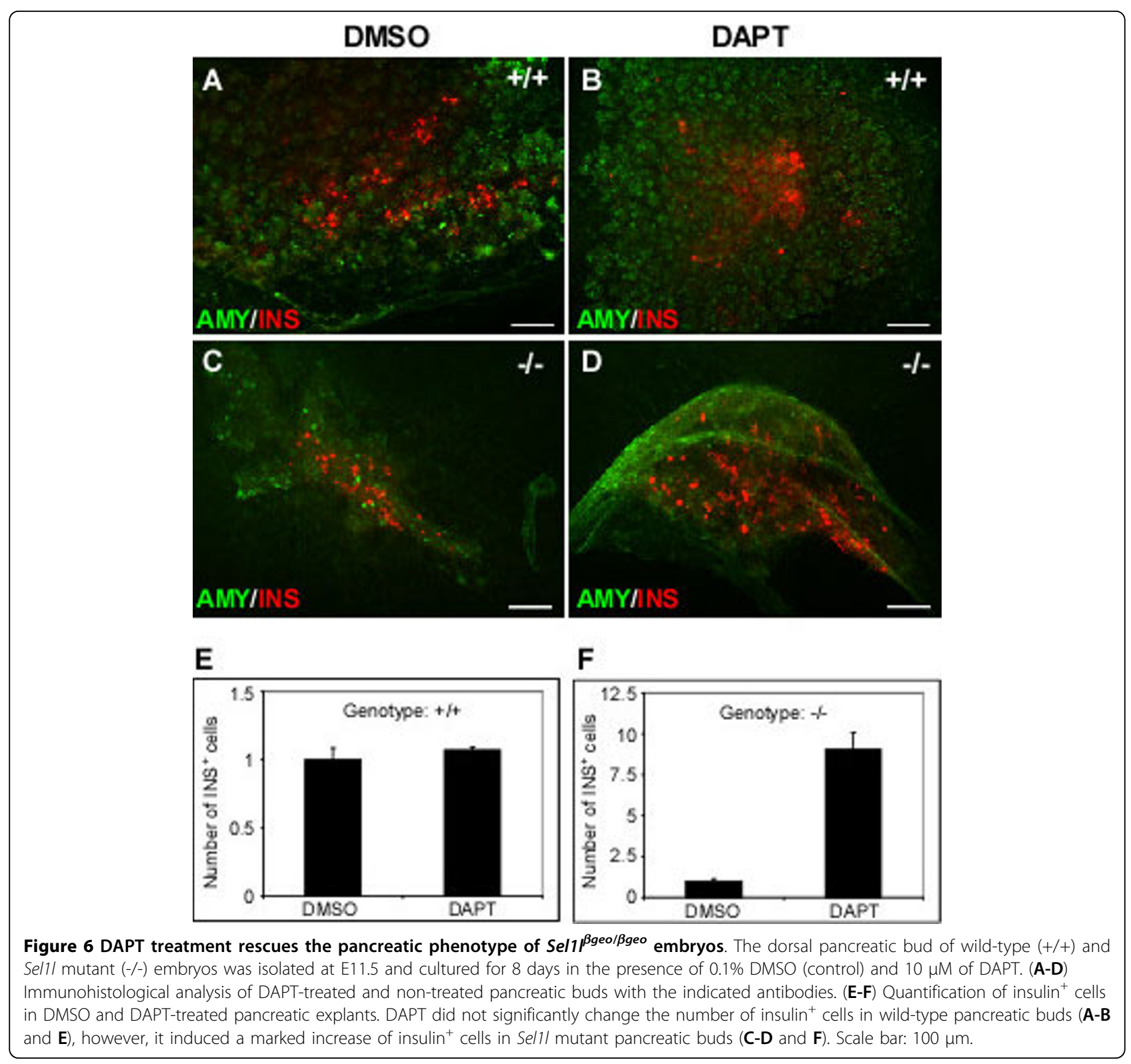

evidence that suggest Sel1l also functions as a negative regulator for Notch signaling, at least in the developing mouse pancreas. First, the observed pancreatic phenotype in Sel1l mutant embryos show remarkable similarity to the pancreatic phenotype of transgenic vertebrate embryos over-expressing the Notch intracellular domain, the constitutively active form of Notch $[26,28,50]$. Second, we show that treatment of cultured Sel1l-deficient pancreatic epithelium with DAPT, a potent Notch signaling inhibitor, partially rescues the growth and differentiation defects of Sel1l-deficient pancreatic epithelium (Fig. 6D and 6F).

How does SEL1L negatively control Notch signaling in the developing mammalian pancreas is the focus of our current investigation. Genetic and biochemical evidences from other organisms such as Drsophila and C. elegans suggest that Notch signaling is regulated at multiple levels and through multiple mechanisms, including stochastical gene expression [34], selective ligand-receptor interactions [35], intracellular trafficking [37], and stability of NICD, the active form of Notch receptors [30]. Given the previous finding that mammalian NICD are ubiquitinated and degraded through both the proteasomal and lysosomal systems $[55,56]$, it is conceivable that Sel11 may be directly or indirectly involved in control of NICD degradation. In this regard, it is noteworthy to mention that recent biochemical data from our laboratory indicate that NICD has significantly higher protein stability in Sel1l-deficient MEF cells (data not shown). 
Further proof of this hypothesis will require a detailed biochemical analysis of SEL1L and Notch interactions.

Biochemical evidences from other laboratories have also underscored the importance of Sel1l in maintaining ER homeostasis. SEL1L interacts with the E3 ubiquitin ligase HRD1 to facilitate the dislocation of unfolded or misfolded proteins from the ER lumen into the cytosol for degradation [48,49,57-60]. Consistent with this, we have recently shown that homozygous Sel1l mutant mice develop systemic ER stress. The impact of ER stress on pancreatic epithelial proliferation and differentiation remains undefined from the current study. However, several hypothetical roles of ER stress in pancreatic organ development and growth can be proposed. First, ER stress affects global gene expression. Upon ER stress, translation is generally down to reduce production of ER client proteins. Concomitantly, transcription of genes encoding ER chaperones and ER-associated degradation (ERAD) machinery is increased. Second, ER stress blocks or reduces protein secretion by mammalian cells. This will directly affect cell signaling and cell functions. Finally, prolonged ER stress activates the signaling pathways leading to apoptosis.

\section{Conclusions}

We report that during mouse pancreatic development, Sel1l is preferentially expressed in the differentiating or differentiated pancreatic epithelial cells. Disruption of Sel1l function results in impaired endocrine lineage formation and delayed exocrine lineage differentiation. Pharmacological suppression of Notch signaling in cultured pancreatic explants partially rescues the endocrine cell differentiation defect. Our data suggest that Sel1l may regulate pancreatic epithelial growth and differentiation by suppressing Notch-mediated signaling.

\section{Methods}

\section{Sel1l gene trap mice}

The chimeric founder mouse used to generate Sel1l gene trap mice was generated by microinjection of a commercially available mouse embryonic stem (ES) cell clone, CA0017, into C57/BL6 blastocysts. This ES cell clone contains a gene trap insertion in the $14^{\text {th }}$ intron of the Sel1l gene. All mice or mouse embryos were genotyped by PCR analysis of tail or toe genomic DNA using the following PCR primers:

\section{F-Sel11: 5'-TGGGACAGAGCGGGCTTGGAAT-3'; R-Sel11: 5'-CACCAGGAGTCAAAGGCATCACTG-3'; R-ßGGeo: 5'ATTCAGGCTGCGCAACTGTTGGG-3'.}

All animal experiments were performed in accordance with the Cornell Animal Care and Use Guidelines.

\section{Histology and immunohistochemistry}

Embryos or pancreatic rudiments were fixed in 4\% paraformaldehyde (PFA) in PBS at $4^{\circ} \mathrm{C}$ for 12 hours or longer. PFA-fixed specimens were equilibrated in $30 \%$ sucrose in PBS at $4^{\circ} \mathrm{C}$ and embedded in O.C.T. Sections were cut at $10 \mu \mathrm{m}$ and immunostained essentially as described $[61,62]$. Briefly, tissue sections were permeabilized with $0.2 \%$ Triton X-100 in PBS for 20 min, washed 3 times in $0.1 \%$ Triton $\mathrm{X}-100$ in PBS and 3 times in PBS. The permeabilized sections were pre-incubated with $5 \%$ normal donkey serum and $1 \%$ BSA in PBS at room temperature for at least 1 hour, followed by incubation in the same solution with primary antibodies at $4^{\circ} \mathrm{C}$ overnight. The antibody-bound sections were then washed three times in $0.1 \%$ Triton X-100 in PBS, 3 times in PBS and then incubated with secondary antibodies for 1-3 hrs at room temperature. Primary antibodies were diluted in 1\% BSA in PBS as follows: rabbit anti-bGalactosidase (Immunology Consultants Laboratory), 1:500; guinea pig anti-Pdx-1 (Abcam), 1:1000; goat antiPdx1 (Abcam), 1:1000; guinea pig anti-insulin (Linco), 1:1000; rabbit anti-glucagon (Covance), 1:500; goat antiglucagon (AbD Serotec), 1:200; rabbit anti-Sox9 (gift from Dr. Michael Wegner), 1:1000; rabbit anti-Ptf1a (gift from Dr. Helena Edlund), 1:1000); and rabbit antiamylase (Sigma), 1:500. Secondary antibodies were diluted in 1\% BSA in PBS as follows: donkey anti-rabbit IgG conjugated to Cy3 (Jackson Immuno Research), 1:1000; donkey anti-guinea pig IgG conjugated to Cy2 (Jackson Immuno Research), 1:500. Fluorescence images were acquired using an Axiovert 40 microscope (Zeiss) equipped with an AxioCam camera.

\section{Pancreatic morphometry and cell counting}

Quantitative morphometry of the developing pancreas and cell counting were performed essentially as described [11]. Briefly, pancreata of age-matched wild-type and Sell $l^{\beta g e o / \beta g e o}$ embryos were sectioned through and every fifth section was immunostained with anti-PDX1 or coimmunostained with anti-PDX1 and anti-Glucagon. Quantification of pancreatic epithelium area $\left(\mathrm{PDX1}^{+}\right.$ area) was performed using AxioVision Imaging analysis software (version 4.6.3). The data were presented as averages $\pm \operatorname{SEM}\left(\mu \mathrm{m}^{2}\right)$ of three independent pancreata. Statistical analysis was performed using the Student's two-sample $t$ test and significance is regarded as $\mathrm{p} \leq 0.05$.

\section{Pancreatic organ culture and DAPT treatment}

Sel1 $l^{+/ \beta g e o}$ mice were intercrossed to generate embryos of defined genotypes: wild-type $\left(\mathrm{Selll}^{+/+}\right)$, heterozygous

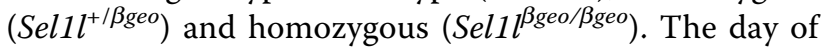
the vaginal plug was taken as embryonic (E) day 0.5. Pancreatic bud dissection and culture were performed 
essentially as described [63]. Briefly, E11.5 embryos were decapitated and the dorsal pancreatic rudiment together with surrounding mesenchymal tissue was removed in PBS. The dissected pancreatic buds were rinsed once in culture medium, transferred to a fibronectin-coated 8well LabTek chamber slide with the epithelial side touching the bottom of the slide and cultured for 72 hours in basal medium with Earle's salts (Gibco 21010046), $1 \times$ glutamine, $10 \%$ FBS and antibiotics. The cultured pancreatic rudiments were fixed individually for 30 minutes in $4 \%$ paraformaldehyde in PBS for immunohistological analysis. DAPT treatment of cultured pancreatic explants was performed essentially as described [64]. DAPT stock solution was prepared at a concentration of $10 \mathrm{mM}$ in DMSO and was aliquoted into single-usage aliquots. For Notch inhibition experiments, pancreatic buds were cultured in complete medium containing $10 \mu \mathrm{M}$ of DAPT. Control pancreatic buds were cultured in complete medium containing $0.1 \%$ DMSO. The DMSO or DAPT-treated pancreatic buds were briefly fixed and analyzed by immunostaining.

Additional file 1: Characterization of the gene trap allele in Sel1I. (A) Schematic representation of the wild-type $\left(\right.$ Sel1/ $\left.1^{+}\right)$and gene trap $\left(\mathrm{Sel} / \mathrm{N}^{\beta \mathrm{geo}}\right)$ allele in Sel11. Solid black rectangles represent exons; the open box in the Sel11- allele represents the gene trap insertion. Splicing events are indicated by dashed lines. (B) PCR analysis of genomic DNAs from wild-type (WT), heterozygous (HT) and mutant (MU) mice using the indicated Sel1/ and $\beta$ geo-specific primers (arrows). The data confirm the presence of a gene trap insertion in intron 14. (C) RT-PCR analysis of RNAs from E12.5 WT, HT and MU embryos using the indicated Sel1I and $\beta$ geo-specific primers (arrows). The data indicate that the gene trap allele in Sel1/ completely blocks RNA splicing between exon 14 and 15, resulting in a truncated Sel11 transcript fused in frame with $\beta$ geo transcript. (D) Schematic representation of the full-length (FL) (top) and deletion mutant (DM) SEL1L peptides generated from the wild-type and gene trap Sel11 alleles. Key protein domains are shown in colored boxes. Numbers represent amino acid positions. The gene trap allele in Sel11 generates a fusion protein containing the N-terminal 465 amino acids of SEL $1 \mathrm{~L}$ and $\beta$ geo. The mutant peptide lacks the Hrd3-like motif, the transmembrane domain, the proline-rich domain and 4 SEL1L-like repeats.

Click here for file

[http://www.biomedcentral.com/content/supplementary/1471-213X-1019-S1.PDF ]

Additional file 2: Sel1/-deficient pancreatic epithelium exhibits impaired growth, branching morphology and differentiation ex

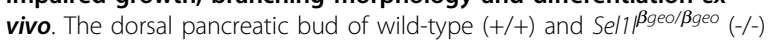
embryos were isolated at E11.5 and cultured as described in Materials and Methods. Cultured pancreatic explants were immunostained with the indicated antibodies. Sel/1-deficient pancreatic epithelium grows poorly and displays impaired branching morphology (B and $\mathbf{D})$ ), as compared to wild-type control explants (A and $\mathbf{C}$ ). Scale bar: $100 \mu \mathrm{m}$. Click here for file

[http://www.biomedcentral.com/content/supplementary/1471-213X-1019-S2.TIFF ]

\section{Acknowledgements}

We thank Dr. Patrick Biggs (Sanger Institute, UK) for generously providing the CA0017 mouse ES cell line; Drs. Raymond MacDonald (The University of Texas Southwestern Medical Center), Chyio Shiota and George Gittes (The
University of Pittsburgh School of Medicine) for useful information about pancreatic explants culture; Drs. Yves Boisclair and Bruce Currie (Cornell University) for stimulating discussions and critical comments during preparation of the manuscript. The financial support for this research project was provided by the College of Agricultural and Life science, Cornell University to QL.

\section{Author details}

${ }^{1}$ Department of Animal Science, College of Agriculture and Life Sciences, Cornell University, Ithaca, NY 14850, USA. ${ }^{2}$ Department of Biomedical Sciences, College of Veterinary Medicine, Cornell University, Ithaca, NY 14850, USA.

\section{Authors' contributions}

$\mathrm{QL}$ designed the study and prepared the manuscript. RM and JS generated the gene trap mice. SL performed the immunohistochemistry, pancreatic bud culture and DAPT rescue experiments. AF maintained the gene trap mice and performed genotyping analysis. All authors reviewed and approved the manuscript.

Received: 17 August 2009

Accepted: 19 February 2010 Published: 19 February 2010

\section{References}

1. Slack JM: Developmental biology of the pancreas. Development 1995, 121:1569-80.

2. Jorgensen MC, Ahnfelt-Ronne J, Hald J, Madsen OD, Serup P, HecksherSorensen J: An illustrated review of early pancreas development in the mouse. Endocr Rev 2007, 28:685-705.

3. Kim SK, MacDonald RJ: Signaling and transcriptional control of pancreatic organogenesis. Curr Opin Genet Dev 2002, 12:540-7.

4. Gu G, Dubauskaite J, Melton DA: Direct evidence for the pancreatic lineage NGN3+ cells are islet progenitors and are distinct from duct progenitors. Development 2002, 129:2447-57.

5. Pictet RL, Clark WR, Williams RH, Rutter WJ: An ultrastructural analysis of the developing embryonic pancreas. Dev Biol 1972, 29:436-67.

6. Ahlgren $U$, Jonsson J, Edlund $H$ : The morphogenesis of the pancreatic mesenchyme is uncoupled from that of the pancreatic epithelium in IPF1/PDX1-deficient mice. Development 1996, 122:1409-16.

7. Offield MF, Jetton TL, Labosky PA, Ray M, Stein RW, Magnuson MA, Hogan BL, Wright CV: PDX-1 is required for pancreatic outgrowth and differentiation of the rostral duodenum. Development 1996, 122:983-95.

8. Kawaguchi Y, Cooper B, Gannon M, Ray M, MacDonald RJ, Wright CV: The role of the transcriptional regulator Ptf1a in converting intestinal to pancreatic progenitors. Nat Genet 2002, 32:128-34.

9. Krapp A, Knofler M, Frutiger S, Hughes GJ, Hagenbuchle O, Wellauer PK: The p48 DNA-binding subunit of transcription factor PTF1 is a new exocrine pancreas-specific basic helix-loop-helix protein. Embo J 1996, 15:4317-29.

10. Lynn FC, Smith SB, Wilson ME, Yang KY, Nekrep N, German MS: Sox9 coordinates a transcriptional network in pancreatic progenitor cells. ProC Natl Acad Sci USA 2007, 104:10500-5.

11. Seymour PA, Freude KK, Tran MN, Mayes EE, Jensen J, Kist R, Scherer G, Sander M: SOX9 is required for maintenance of the pancreatic progenitor cell pool. Proc Natl Acad Sci USA 2007, 104:1865-70.

12. Gradwohl G, Dierich A, LeMeur M, Guillemot F: neurogenin3 is required for the development of the four endocrine cell lineages of the pancreas. Proc Natl Acad Sci USA 2000, 97:1607-11.

13. Apelqvist A, Li H, Sommer L, Beatus P, Anderson DJ, Honjo T, Hrabe de Angelis $\mathrm{M}$, Lendahl $\mathrm{U}$, Edlund $\mathrm{H}$ : Notch signalling controls pancreatic cell differentiation. Nature 1999, 400:877-81.

14. Naya FJ, Huang HP, Qiu Y, Mutoh H, DeMayo FJ, Leiter AB, Tsai MJ: Diabetes defective pancreatic morphogenesis and abnormal enteroendocrine differentiation in BETA2/neuroD-deficient mice. Genes Dev 1997, 11:2323-34.

15. Naya FJ, Stellrecht CM, Tsai MJ: Tissue-specific regulation of the insulin gene by a novel basic helix-loop-helix transcription factor. Genes Dev 1995, 9:1009-19.

16. Sosa-Pineda B, Chowdhury K, Torres M, Oliver G, Gruss P: The Pax4 gene is essential for differentiation of insulin-producing beta cells in the mammalian pancreas. Nature 1997, 386:399-402. 
17. St-Onge L, Sosa-Pineda B, Chowdhury K, Mansouri A, Gruss P: Pax6 is required for differentiation of glucagon-producing alpha-cells in mouse pancreas. Nature 1997, 387:406-9.

18. Sussel L, Kalamaras J, Hartigan-O'Connor DJ, Meneses JJ, Pedersen RA, Rubenstein JL, German MS: Mice lacking the homeodomain transcription factor Nkx2.2 have diabetes due to arrested differentiation of pancreatic beta cells. Development 1998, 125:2213-21.

19. Sander M, Sussel L, Conners J, Scheel D, Kalamaras J, Dela Cruz F, Schwitzgebel V, Hayes-Jordan A, German M: Homeobox gene Nkx6.1 lies downstream of Nkx2.2 in the major pathway of beta-cell formation in the pancreas. Development 2000, 127:5533-40.

20. Collombat P, Mansouri A, Hecksher-Sorensen J, Serup P, Krull J, Gradwohl G, Gruss P: Opposing actions of Arx and Pax4 in endocrine pancreas development. Genes Dev 2003, 17:2591-603.

21. Ahlgren U, Pfaff $S L$, Jessell TM, Edlund T, Edlund H: Independent requirement for ISL1 in formation of pancreatic mesenchyme and islet cells. Nature 1997, 385:257-60.

22. Gierl MS, Karoulias N, Wende $H$, Strehle M, Birchmeier C: The zinc-finger factor Insm1 (IA-1) is essential for the development of pancreatic beta cells and intestinal endocrine cells. Genes Dev 2006, 20:2465-78.

23. Johansson KA, Dursun U, Jordan N, Gu G, Beermann F, Gradwohl G, GrapinBotton A: Temporal control of neurogenin3 activity in pancreas progenitors reveals competence windows for the generation of different endocrine cell types. Dev Cell 2007, 12:457-65.

24. Jensen J, Pedersen EE, Galante P, Hald J, Heller RS, Ishibashi M, Kageyama R, Guillemot F, Serup P, Madsen OD: Control of endodermal endocrine development by Hes-1. Nat Genet 2000, 24:36-44.

25. Fujikura J, Hosoda K, Iwakura H, Tomita T, Noguchi M, Masuzaki H, Tanigaki K, Yabe D, Honjo T, Nakao K: Notch/Rbp-j signaling prevents premature endocrine and ductal cell differentiation in the pancreas. Cell Metab 2006, 3:59-65.

26. Esni F, Ghosh B, Biankin AV, Lin JW, Albert MA, Yu X, MacDonald RJ, Civin Cl, Real FX, Pack MA, Ball DW, Leach SD: Notch inhibits Ptf1 function and acinar cell differentiation in developing mouse and zebrafish pancreas. Development 2004, 131:4213-24

27. Hald J, Hjorth JP, German MS, Madsen OD, Serup P, Jensen J: Activated Notch1 prevents differentiation of pancreatic acinar cells and attenuate endocrine development. Dev Biol 2003, 260:426-37.

28. Murtaugh LC, Stanger BZ, Kwan KM, Melton DA: Notch signaling controls multiple steps of pancreatic differentiation. Proc Natl Acad Sci USA 2003, 100:14920-5.

29. Greenwald I: LIN-12/Notch signaling: lessons from worms and flies. Genes Dev 1998, 12:1751-62.

30. Baron M, Aslam H, Flasza M, Fostier M, Higgs JE, Mazaleyrat SL, Wilkin MB: Multiple levels of Notch signal regulation (review). Mol Membr Biol 2002, 19:27-38.

31. Justice NJ, Jan YN: Variations on the Notch pathway in neural development. Curr Opin Neurobiol 2002, 12:64-70.

32. Cormier S, Vandormael-Pournin S, Babinet C, Cohen-Tannoudji M: Developmental expression of the Notch signaling pathway genes during mouse preimplantation development. Gene Expr Patterns 2004, 4:713-7.

33. Haddon C, Smithers L, Schneider-Maunoury S, Coche T, Henrique D, Lewis J: Multiple delta genes and lateral inhibition in zebrafish primary neurogenesis. Development 1998, 125:359-70.

34. Kaern M, Elston TC, Blake WJ, Collins JJ: Stochasticity in gene expression: from theories to phenotypes. Nat Rev Genet 2005, 6:451-64.

35. Fleming RJ, Gu Y, Hukriede NA: Serrate-mediated activation of Notch is specifically blocked by the product of the gene fringe in the dorsal compartment of the Drosophila wing imaginal disc. Development 1997, 124:2973-81.

36. Lawrence N, Klein T, Brennan K, Martinez Arias A: Structural requirements for notch signalling with delta and serrate during the development and patterning of the wing disc of Drosophila. Development 2000, 127:3185-95

37. Le Borgne $\mathrm{R}$, Bardin A, Schweisguth F: The roles of receptor and ligand endocytosis in regulating Notch signaling. Development 2005, 132:1751-62.

38. Lai EC: Protein degradation: four E3s for the notch pathway. Curr Biol 2002, 12:R74-8.
39. Biunno I, Castiglioni B, Rogozin IB, DeBellis G, Malferrari G, Cattaneo M: Cross-species conservation of SEL1L, a human pancreas-specific expressing gene. Omics 2002, 6:187-98.

40. Biunno I, Appierto V, Cattaneo M, Leone BE, Balzano G, Socci C, Saccone S, Letizia A, Della Valle G, Sgaramella V: Isolation of a pancreas-specific gene located on human chromosome 14q31: expression analysis in human pancreatic ductal carcinomas. Genomics 1997, 46:284-6.

41. Donoviel DB, Donoviel MS, Fan E, Hadjantonakis A, Bernstein A: Cloning and characterization of Sel-1l, a murine homolog of the $C$. elegans sel-1 gene. Mech Dev 1998, 78:203-7.

42. Su Al, Cooke MP, Ching KA, Hakak Y, Walker JR, Wiltshire T, Orth AP, Vega RG, Sapinoso LM, Moqrich A, Patapoutian A, Hampton GM, Schultz PG, Hogenesch JB: Large-scale analysis of the human and mouse transcriptomes. Proc Natl Acad Sci USA 2002, 99:4465-70.

43. Donoviel DB, Bernstein A: SEL-1L maps to human chromosome 14, near the insulin-dependent diabetes mellitus locus 11. Genomics 1999, 56:232-3.

44. Sundaram $M$, Greenwald I: Suppressors of a lin-12 hypomorph define genes that interact with both lin-12 and glp-1 in Caenorhabditis elegans. Genetics 1993, 135:765-83.

45. Grant B, Greenwald I: The Caenorhabditis elegans sel-1 gene, a negative regulator of lin-12 and glp-1, encodes a predicted extracellular protein Genetics 1996, 143:237-47.

46. Grant B, Greenwald I: Structure, function, and expression of SEL-1, a negative regulator of LIN-12 and GLP-1 in C. elegans. Development 1997, 124:637-44.

47. Rooman I, De Medts N, Baeyens L, Lardon J, De Breuck S, Heimberg H, Bouwens $L$ : Expression of the Notch signaling pathway and effect on exocrine cell proliferation in adult rat pancreas. Am J Pathol 2006, 169:1206-14

48. Hosokawa N, Wada I, Nagasawa K, Moriyama T, Okawa K, Nagata K: Human XTP3-B forms an endoplasmic reticulum quality control scaffold with the HRD1-SEL1L ubiquitin ligase complex and BiP. J Biol Chem 2008, 283:20914-24.

49. Mueller B, Lilley BN, Ploegh HL: SEL1L, the homologue of yeast Hrd3p, is involved in protein dislocation from the mammalian ER. J Cell Biol 2006, 175:261-70.

50. Zecchin E, Filippi A, Biemar F, Tiso N, Pauls S, Ellertsdottir E, Gnugge L, Bortolussi M, Driever W, Argenton F: Distinct delta and jagged genes control sequential segregation of pancreatic cell types from precursor pools in zebrafish. Dev Biol 2007, 301:192-204

51. Wessells NK, Evans J: Ultrastructural studies of early morphogenesis and cytodifferentiation in the embryonic mammalian pancreas. Dev Biol 1968, 17:413-46

52. Rall $L B$, Pictet $R L$, Williams $R H$, Rutter WJ: Early differentiation of glucagonproducing cells in embryonic pancreas: a possible developmental role for glucagon. Proc Natl Acad Sci USA 1973, 70:3478-82.

53. Sundaram $M$, Greenwald I: Genetic and phenotypic studies of hypomorphic lin-12 mutants in Caenorhabditis elegans. Genetics 1993 135:755-63.

54. Chiaramonte R, Calzavara E, Basile A, Comi P, Sherbet GV: Notch signal transduction is not regulated by SEL1L in leukaemia and lymphoma cells in culture. Anticancer Res 2002, 22:4211-4.

55. Mukherjee A, Veraksa A, Bauer A, Rosse C, Camonis J, Artavanis-Tsakonas S: Regulation of Notch signalling by non-visual beta-arrestin. Nat Cell Biol 2005, 7:1191-201.

56. Chastagner $P$, Israel A, Brou C: AIP4/Itch regulates Notch receptor degradation in the absence of ligand. PLOS ONE 2008, 3:e2735.

57. Cattaneo M, Otsu M, Fagioli C, Martino S, Lotti LV, Sitia R, Biunno I: SEL1L and HRD1 are involved in the degradation of unassembled secretory lgmu chains. J Cell Physiol 2008, 215:794-802.

58. Cormier JH, Tamura T, Sunryd JC, Hebert DN: EDEM1 recognition and delivery of misfolded proteins to the SEL1L-containing ERAD complex. Mol Cell 2009, 34:627-33.

59. Mueller B, Klemm EJ, Spooner E, Claessen JH, Ploegh HL: SEL1L nucleates a protein complex required for dislocation of misfolded glycoproteins. Proc Natl Acad Sci USA 2008, 105:12325-30.

60. Oresic K, Mueller B, Tortorella D: Cln6 mutants associated with neuronal ceroid lipofuscinosis are degraded in a proteasome-dependent manner. Biosci Rep 2009, 29:173-81. 
61. Burlison JS, Long Q, Fujitani Y, Wright CV, Magnuson MA: Pdx-1 and Ptf1a concurrently determine fate specification of pancreatic multipotent progenitor cells. Dev Biol 2008, 316:74-86

62. Matsuoka TA, Zhao L, Artner I, Jarrett HW, Friedman D, Means A, Stein R: Members of the large Maf transcription family regulate insulin gene transcription in islet beta cells. Mol Cell Biol 2003, 23:6049-62.

63. Percival AC, Slack JM: Analysis of pancreatic development using a cell lineage label. Exp Cell Res 1999, 247:123-32.

64. Cheng HT, Miner JH, Lin M, Tansey MG, Roth K, Kopan R: Gamma-secretase activity is dispensable for mesenchyme-to-epithelium transition but required for podocyte and proximal tubule formation in developing mouse kidney. Development 2003, 130:5031-42.

doi:10.1186/1471-213X-10-19

Cite this article as: $L i$ et al:: SEL1L deficiency impairs growth and differentiation of pancreatic epithelial cells. BMC Developmental Biology 2010 10:19.

\section{Submit your next manuscript to BioMed Central} and take full advantage of:

- Convenient online submission

- Thorough peer review

- No space constraints or color figure charges

- Immediate publication on acceptance

- Inclusion in PubMed, CAS, Scopus and Google Scholar

- Research which is freely available for redistribution

Submit your manuscript at www.biomedcentral.com/submit 